\title{
Aspectos tecnológicos na produção de farinha de polpa de pequi
}

\section{Technological aspects in the production of pequi pulp flour}

\author{
Priscilla Narciso Justi ${ }^{1}$; Sueli Marie Ohata ${ }^{2}$; Cândida Aparecida Leite Kassuya ${ }^{3}$; Maria Lígia Rodrigues Macedo ${ }^{4}$ Eliana \\ Janet Sanjinez Argandoña ${ }^{5}$
}

Resumo: Objetivou-se avaliar o efeito da temperatura de secagem nas características nutricionais da farinha da polpa de pequi. A secagem da polpa de pequi foi realizada em um secador de bandejas com fluxo de ar quente a $0,5 \mathrm{~m} \mathrm{~s}^{-1} \mathrm{em}$ três temperaturas 55,65 e $70{ }^{\circ} \mathrm{C}$. As cinéticas de secagem foram avaliadas ajustando-se os dados experimentais através de sete modelos matemáticos por regressão não linear. Determinaram-se a difusividade efetiva e a energia de ativação requerida para a evaporação da umidade do produto. Os resultados mostraram redução do tempo de secagem com o aumento da temperatura do processo. Os ajustes dos modelos foram avaliados em função dos valores de $\mathrm{R}^{2}$ e ERM. Todos os modelos apresentaram bons indicadores estatísticos, entretanto, o modelo que representou os melhores valores da cinética de secagem da polpa de pequi, para as condições estudadas, foi o de Page. Os valores da difusividade efetiva nas condições de temperatura estudadas estão entre 4,04 a $5,57 \times 10^{-10} \mathrm{~m}^{2} \mathrm{~s}^{-1}$ e a energia de ativação foi de 2,03 x $10^{4} \mathrm{~J} \mathrm{~mol}^{-1}$. O processo de secagem favoreceu na concentração de alguns nutrientes como carboidratos e proteínas. A desidratação a $55^{\circ} \mathrm{C}$ forneceu um produto com melhor aparência visual entre as temperaturas estudadas.

Palavras-chave: Caryocar brasiliense; Cinéticas de secagem; Difusividade efetiva.

\begin{abstract}
The objective of this study was to evaluate the effect of drying temperature on the nutritional characteristics of pequi pulp flour.The dehydrated pequi pulp was accomplished in a dryer tray with hot air flow of $0,5 \mathrm{~m} \mathrm{~s}^{-1}$ at three temperatures 55 , 65 and $70{ }^{\circ} \mathrm{C}$. The drying kinetic were evaluated by adjusting experimental data using mathematical models by seven nonlinear regression. It was determined the effective diffusivity and energy activation required for the evaporation of the moisture from the product. The results showed a reduction of the drying time with an increasing process of the temperature. The adjustment of the models was evaluated according to the values of $\mathrm{R}^{2}$ and ERM. All models showed good adjustments, however, the Page model was the one that best values of the drying kinetics of pequi pulp for the conditions studied. The effective diffusivity values in the temperature studied are from 4,04 to $5,57 \times 10^{-10} \mathrm{~m}^{2} \mathrm{~s}^{-1}$ and the activation energy was $2,03 \mathrm{x}$ $10^{4} \mathrm{~J} \mathrm{~mol}{ }^{-1}$. Drying process have favored on the concentration of some nutrients, such as carbohydrates and proteins. Dehydration at $55^{\circ} \mathrm{C}$ resulted in a product with better visual appearence among the studied temperatures.
\end{abstract}

Keywords: Caryocar brasiliense; Drying kinetic; Effective diffusivity.

\footnotetext{
*Autor para correspondência

Recebido para publicação em 15/06/2017; aprovado em 04/09/2017

${ }^{1}$ Doutora em Saúde e Desenvolvimento na Região Centro Oeste, Universidade Federal da Grande Dourados, Dourados - MS, priscillajusti@ufgd.edu.br

${ }^{2}$ Docente da Universidade Federal da Grande Dourados, Faculdade de Engenharia, Dourados - MS, sueliohata@ufgd.edu.br

${ }^{3}$ Docente da Universidade Federal da Grande Dourados, Faculdade de Ciências da Saúde, Dourados - MS, candida2005@ gmail.com

${ }^{4}$ Docente da Universidade Federal de Mato Grosso do Sul, Faculdade de Ciências Médicas, Campo Grande - MS, bioplant@ terra.com.br

${ }^{5}$ Docente da Universidade Federal da Grande Dourados, Faculdade de Engenharia, Dourados - MS, elianaargandona@ufgd.edu.br
} 


\section{INTRODUÇÃO}

A região do Cerrado brasileiro é a segunda biodiversidade da América do Sul, correspondendo a 23\% da área total do País superada apenas pela Amazônia, estando presente em 13 estados brasileiros e no Distrito Federal (QUEIROZ, 2009). Este bioma oferece às suas populações uma grande variedade de produtos que podem ser importantes na promoção de meios de vida sustentáveis, onde a geração de renda e qualidade de vida esteja em consonância com a conservação de recursos naturais (MOURA et al., 2013).

$\mathrm{Na}$ vegetação do Cerrado as fruteiras nativas ocupam lugar de destaque. Entre essas espécies a Caryocar brasiliense representa importante fonte de alimento e de renda de famílias (LOPES et al., 2012). A comercialização do pequi baseia-se na venda de caroços, polpa em fatias, amêndoas, óleos da polpa e da amêndoa, conservas e licores. É um fruto que possui curto período de vida útil na forma in natura, devendo ser consumido rapidamente ou conservado sob congelamento durante a sua entressafra.

Diante disso, técnicas alternativas que favoreçam a disponibilidade da polpa vêm sendo utilizadas visando rapidez e praticidade ao consumidor, assim como, a manutenção das propriedades nutritivas presentes no alimento após seu processamento. No entanto, ainda são necessários estudos que contribuam para o melhor aproveitamento dos frutos na obtenção de novos gêneros alimentícios que possam promover a valorização das plantas naturais do cerrado.

A secagem é uma técnica de conservação que se baseia no fato de que tanto os microrganismos, enzimas e mecanismo metabólico, necessitam de certa quantidade de água para suas atividades. Com a redução da água, consequentemente serão reduzidas a atividade de água, a velocidade das reações químicas no produto e o crescimento de microrganismos (OLIVEIRA et al., 2015).

$\mathrm{Na}$ secagem da maioria dos produtos de origem agrícola, o mecanismo de transporte de umidade mais empregado tem sido a difusão líquida, embora existam algumas considerações para sua aplicação, como: redução do volume desprezado, inexistência do efeito de capilaridade, equilíbrio térmico instantâneo com o ar e os efeitos da transferência de energia e massa de um corpo, admitidos como desprezíveis (GONELI et al., 2007).

Os modelos teóricos que descrevem a taxa decrescente de secagem de um sólido consideram, geralmente, como mecanismo principal, a difusão baseada na segunda Lei de Fick, que expressa que o fluxo de massa por unidade de área é proporcional ao gradiente de concentração de água (CELMA et al., 2009).

Estudos sobre a cinética de secagem em camada delgada dão ênfase aos modelos semiteóricos que se baseiam na Lei de Newton para resfriamento, aplicada à transferência de massa. Entre os modelos semiteóricos, o modelo de Dois Termos, de Henderson e Pabis e o de Page têm sido utilizados (SIQUEIRA et al., 2013).

Embora a secagem favoreça à conservação dos alimentos, as etapas durante a desidratação podem ocasionar a destruição de nutrientes. Contudo, o emprego da técnica de desidratação, confere outras formas de utilização e preservação da polpa, além de aumentar a vida útil do produto e promover a elaboração de alimentos diferenciados (MACHADO et al., 2010)
Ante o exposto objetivou-se avaliar o efeito da temperatura de secagem nas características nutricionais da farinha da polpa de pequi.

\section{MATERIAL E MÉTODOS}

Para a realização deste estudo os frutos de pequi (Caryocar brasiliense Camb.) foram adquiridos no assentamento Lagoa Grande em Dourados, Mato Grosso do Sul, selecionados de acordo com seu estado de maturação e sanidade fisiológica, em seguida transportados para o laboratório de Tecnologia de Alimentos da Faculdade de Engenharia da Universidade Federal da Grande Dourados.

Os frutos de pequi foram lavados em água corrente, sanitizados em $200 \mathrm{mg}$ de solução de dicloroisocianurato de sódio dehidratado $\mathrm{L}^{-1}$ (Sumaveg $\left.{ }^{\circledR}\right)$, imersos em água a $70{ }^{\circ} \mathrm{C}$ por cinco minutos e postos para drenar o excesso. Em seguida, os pequis foram despolpados manualmente com faca de aço inoxidável, cortados em pedaços, acondicionados em embalagens de polietileno e congelados a $-18{ }^{\circ} \mathrm{C}$ até a realização das análises de umidade, resíduo mineral, proteínas, lipídios e cor.

No processo de secagem a polpa de pequi foi previamente descongelada a $10^{\circ} \mathrm{C}$ por $24 \mathrm{~h}$ e em seguida, aproximadamente $22 \mathrm{~g}$ de amostra, foram colocadas em bandejas de aço inoxidável medindo 43,96 $\mathrm{cm}$ de comprimento e $2,0 \mathrm{~cm}$ de profundidade formando uma camada delgada homogênea de espessura aproximada de 5 $\mathrm{mm}$ e desidratada em um secador de leito fixo Cienlab CE$220 / 630$ com velocidade do ar quente a $0,5 \mathrm{~m} \mathrm{~s}^{-1}$, medidos com anemômetro digital. As cinéticas de secagem foram realizadas a 55,65 e $70{ }^{\circ} \mathrm{C}$. Durante a operação de secagem realizaram-se pesagens periódicas em balança analítica Bel M124Ai em intervalos regulares de $10 \mathrm{~min}$ na primeira hora e de 30 min nas horas subsequentes, até que o teor de água da polpa atingisse a umidade de equilíbrio. Após a secagem o material ficou em repouso em dessecador até atingir equilíbrio térmico com o meio e posteriormente triturado em moinho de facas Solab SL32. A farinha obtida foi peneirada em peneiras com abertura de $1,41 \mathrm{~mm}$ com a finalidade de se obter uniformidade na granulometria do material.

A análise do teor de água da polpa fresca e da polpa seca do pequi foi realizada, em triplicata, pelo método gravimétrico em estufa de circulação de ar Quimis Q314M a $70 \pm 1{ }^{\circ} \mathrm{C}$ por $24 \mathrm{~h}$.

Para cada temperatura foram realizadas curvas de secagem expressas como a variação da razão de umidade em função do tempo de secagem (Eq.1) e a taxa de secagem $(\mathrm{dx} / \mathrm{dt})$ calculada pela derivada da umidade em relação ao tempo (Eq.2). As razões da umidade do produto foram calculadas pela Eq.1.

$$
\mathrm{Y}=\frac{\mathrm{U}-\mathrm{U}_{\mathrm{e}}}{\mathrm{U}_{0}-\mathrm{U}_{\mathrm{e}}}
$$

Em que: Y - razão de umidade do produto (adimensional); $\mathrm{U}$ - teor de água do produto (decimal b.s.); $\mathrm{U}_{0}$ - teor de água inicial do produto (decimal b.s.); $\mathrm{U}_{\mathrm{e}}=$ teor de água de equilíbrio do produto (decimal b.s.).

$$
\mathrm{Ts}=\frac{\mathrm{dx}}{\mathrm{dt}}=\frac{\mathrm{U}_{\mathrm{n}-1}-\mathrm{U}_{\mathrm{n}}}{\mathrm{U}_{\mathrm{n}-1}-\mathrm{U}^{\mathrm{n}}}
$$


Em que: $\mathrm{t}$ - tempo (s); $\mathrm{n}$ - número de leituras; $\mathrm{dx} / \mathrm{dt}$ taxa de secagem; $U_{n}$ e $U_{n-1}$ - umidade no tempo n e no tempo $\mathrm{n}-1$.
Para o ajuste dos dados experimentais das cinéticas de secagem foram utilizados os modelos matemáticos apresentados na Tabela 1 .

Tabela 1. Lista de modelos matemáticos empregados no ajuste de cinéticas de secagem.

\begin{tabular}{lcc}
\hline \multicolumn{1}{c}{ Modelo } & Equação & Referências \\
\hline Difusão (lei de Fick) & $\mathrm{y}=\mathrm{a}\left[-\mathrm{c}\left(\frac{\mathrm{t}}{\mathrm{L}^{2}}\right)\right]$ & Diamante e Munro (1991) \\
Dois termos & $\mathrm{Y}=\mathrm{a} \exp \left(-\mathrm{k}_{\mathrm{o}} \cdot \mathrm{t}\right)+\mathrm{bexp}\left(-\mathrm{k}_{1} \cdot \mathrm{t}\right)$ & Ozdemir e Devres (1999) \\
Henderson e Pabis & $\mathrm{Y}=\mathrm{a} \exp (-\mathrm{k} \cdot \mathrm{t})$ & Henderson e Pabis (1961) \\
Logarítmico & $\mathrm{Y}=\mathrm{a} \exp (-\mathrm{k} \cdot \mathrm{t})+\mathrm{c}$ & Yaldiz et al. (2001) \\
Newton & $\mathrm{Y}=\exp (-\mathrm{k} \cdot \mathrm{t})$ & Abe e Afzal (1997) \\
Page & $\mathrm{Y}=\exp \left(-\mathrm{k} \cdot \mathrm{t}^{\mathrm{n}}\right)$ & Page (1949) \\
Wang e Singh & $\mathrm{Y}=\mathrm{at}^{2}+b t+1$ & Wang e Singh (1978)
\end{tabular}

Y-adimensional; a, b, c, k, $\mathrm{k}_{\mathrm{o}}, \mathrm{k}_{1}$ e $\mathrm{n}$ - coeficientes dos modelos; $\mathrm{t}$ - tempo (s); L - espessura da placa (m).

A avaliação da adequação dos modelos empregados na descrição das cinéticas foi feita em função do coeficiente de determinação $\left(\mathrm{R}^{2}\right)$ estimado como o quadrado do coeficiente de correlação linear entre valores preditos e observados para a variável dependente razão de umidade e do erro médio relativo (EMR) calculado a partir das Equações. 3 e 4 . Os modelos que apresentaram EMR $<10 \%$ foram considerados preditivos.

$$
\begin{aligned}
& \mathrm{R}^{2}=\frac{\mathrm{SSr}}{\mathrm{SST}} \\
& \mathrm{EMR}=\frac{100}{\mathrm{n}} \sum_{\mathrm{i}=1}^{\mathrm{n}}\left[\frac{\mathrm{V}-\mathrm{V}_{\mathrm{e}}}{\mathrm{v}}\right]
\end{aligned}
$$

Em que: SSr - soma quadrática devido à regressão; SST - soma quadrática em torno da média; n - número de observações; V - valores observados; $\mathrm{V}_{\mathrm{e}}$ - valores estimados; $\mathrm{z}$ - número de constantes no modelo.

O coeficiente de difusão para as condições de secagem foi calculado ajustando-se o modelo baseado na teoria de difusão líquida aos dados observados. A Equação 5 é a solução analítica para a segunda Lei de Fick nas condições de geometria de placa plana infinita, coeficiente de difusão constante, umidade inicial uniformemente distribuída no interior do produto, resistência externa desprezível, encolhimento desprezível e umidade constante na superfície sendo igual à umidade de equilíbrio (CRANK, 1975).

$$
\mathrm{Y}=\frac{8}{\pi^{2}} \sum \frac{1}{(2 \mathrm{i}+1)^{2}} \exp \left[\frac{-(2 \mathrm{i}-1)^{2} \pi^{2} \mathrm{D}_{\mathrm{ef}} \mathrm{t}}{4 \mathrm{~L}^{2}}\right]
$$

Em que: Y - razão de umidade do produto (adimensional); $\mathrm{D}_{\mathrm{ef}}$ - coeficiente de difusão efetivo $\left(\mathrm{m}^{2} \mathrm{~s}^{-1}\right) ; \mathrm{i}$ - número de termos da série; $\mathrm{t}$ - tempo (s); L - metade da espessura da amostra $(\mathrm{m})$.

A dependência do coeficiente de difusão efetivo com a temperatura foi analisada pela equação de Arrhenius. Eq. 6.

$$
\mathrm{D}_{\mathrm{ef}}=\mathrm{D}_{0} \cdot \exp \left(\frac{-\mathrm{E}_{\mathrm{a}}}{\mathrm{RT}_{\mathrm{a}}}\right)
$$

Em que: $\mathrm{D}_{\mathrm{ef}}-$ coeficiente de difusão efetivo $\left(\mathrm{m}^{2} \mathrm{~s}^{-1}\right) ; \mathrm{D}_{\mathrm{o}}$ - fator pré-exponencial $\left(\mathrm{m}^{2} \mathrm{~s}^{-1}\right) ; \mathrm{E}_{\mathrm{a}}$ - energia de ativação $(\mathrm{J}$ $\left.\mathrm{mol}^{-1}\right) ; \mathrm{R}$ - constante universal dos gases $\left(8,314 \times 10^{3} \mathrm{~J} \mathrm{~mol}^{-1}\right.$ $\left.\mathrm{K}^{-1}\right) ; \mathrm{T}_{\mathrm{a}}$ - temperatura absoluta $\left(\mathrm{K}^{-1}\right)$.

Os coeficientes da expressão de Arrhenius foram linearizados com a aplicação do logaritmo Eq. 7. A energia de ativação foi calculada pela inclinação da reta $\left(\mathrm{E}_{\mathrm{a}} / \mathrm{R}\right)$.

$$
\ln \mathrm{D}_{\mathrm{ef}}=\ln \mathrm{D}_{0}-\frac{\mathrm{E}_{\mathrm{a}}}{\mathrm{R}} \cdot \frac{1}{\mathrm{~T}_{\mathrm{a}}}
$$

Avaliou-se o teor de umidade, resíduo mineral, proteínas e lipídios conforme o método descrito pelo IAL (2008) nas polpas frescas e nas farinhas. O teor de carboidratos foi calculado por diferença. A cor foi determinada em colorimetro Minolta utilizando o espaço de cor CIELAB, onde $\mathrm{L}^{*}$ é a luminosidade, a* coordenada vermelho/verde (+ a indica vermelho e - a indica verde), $b^{*}$ coordenada amarelo/azul (+ b indica amarelo e $-\mathrm{b}$ indica azul). Todas as análises foram realizadas em triplicata a partir de diferentes lotes e os resultados expressos pela média com o desvio padrão.

O delineamento experimental utilizado na secagem foi inteiramente casualizado composto de três tratamentos e três repetições por tratamento.

\section{RESULTADOS E DISCUSSÃO}

A Figura 1. apresenta a cinética de secagem da polpa de pequi a 55,65 e $70{ }^{\circ} \mathrm{C}$ em função da razão de umidade e do tempo. Observa-se que o aumento da temperatura de 65 para $70{ }^{\circ} \mathrm{C}$ pouco influenciou na perda de umidade do produto.

De acordo com a legislação brasileira, a umidade máxima permitida em farinhas é de 15\% (BRASIL, 2005), considerou-se a umidade final do pequi de $10 \%$, como sendo a umidade necessária para manter o produto estável por maior tempo de estocagem. Os tempos de secagem necessários para se obter um produto estável com $10 \%$ de umidade (base úmida) ou $0,16 \mathrm{~kg}_{\text {água }} \mathrm{kg}_{\text {sólido seco }}{ }^{-1}$ (base seca) de acordo com a Figura 1. foram 220, 240 e 300 min para 70,65 e $55^{\circ} \mathrm{C}$ respectivamente. 
Figura 1. Conteúdo de umidade (base seca) em função do tempo de secagem da polpa de pequi desidratada a 55,65 e $70{ }^{\circ} \mathrm{C}$.

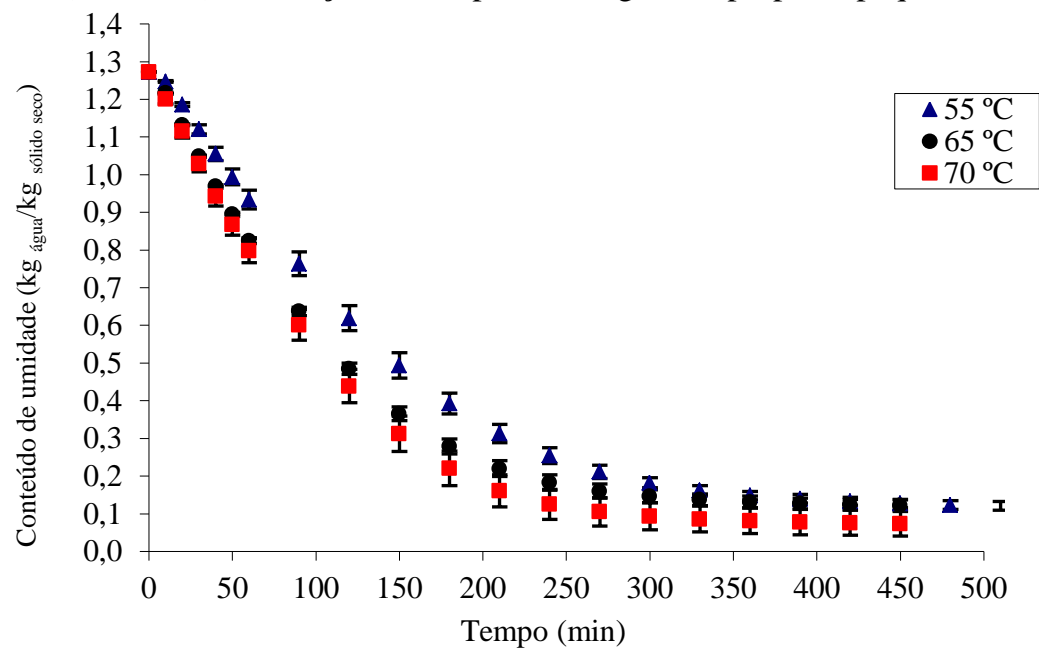

Os valores dos parâmetros obtidos em cada modelo matemático e os parâmetros estatísticos para as diferentes condições de secagem estão apresentados na Tabela 2. Os valores de $\mathrm{R}^{2}$ e ERM variaram de 0,9840 a 0,9999 e 0,4082 a 4,4897 , respectivamente. Isto indica que houve bom ajuste entre os valores observados e os preditos (ERM $<10 \%)$. Portanto, os modelos podem ser utilizados para prever os dados experimentais dentro da faixa de temperatura estudada.

Contudo, o modelo que melhor representou a secagem em camada delgada da polpa de pequi para as condições estudadas foi o modelo de Page $\left(\mathrm{R}^{2}=0,9999\right)$. O modelo de Page também apresentou bom ajuste aos dados experimentais de secagem da polpa de pequi da espécie Coriaceum (SOUSA et al., 2017) e do broto de pera espinhosa (PEREIRA et al.,
2017) ambas secas em estufa nas temperaturas de 50, 60, 70 e $80{ }^{\circ} \mathrm{C}$, apresentando $\mathrm{R}^{2}$ maior que 0,99 .

De acordo com a Tabela 2, observa-se que o parâmetro $k$ no modelo de Page aumentou com o aumento da temperatura e o parâmetro $n$ parece não apresentar dependência da temperatura, evidenciando a alta afinidade do modelo aos dados experimentais da cinética de secagem da polpa de pequi. De acordo com Azzouz et al. (2002) o parâmetro k é influenciado pela temperatura e umidade inicial do produto e o parâmetro $n$ pela velocidade do ar de secagem e da umidade inicial. Com base nos valores apresentados realizou-se o ajuste dos dados. A Figura 2. mostra o desempenho dessa equação na estimativa da secagem a 55,65 e $70{ }^{\circ} \mathrm{C}$.

Tabela 2. Parâmetros obtidos dos modelos, coeficiente de determinação $\left(\mathrm{R}^{2}\right)$ e erro relativo médio (ERM), calculados para verificação do ajuste dos modelos matemáticos aos valores experimentais da secagem da polpa de pequi obtidos nas temperaturas de 55,65 e $70{ }^{\circ} \mathrm{C}$.

\begin{tabular}{|c|c|c|c|c|c|c|c|}
\hline \multirow[t]{2}{*}{ Modelos matemático } & \multicolumn{5}{|c|}{ Parâmetros dos modelos } & \multicolumn{2}{|c|}{ Parâmetros estatísticos } \\
\hline & $\mathrm{T}\left({ }^{\circ} \mathrm{C}\right)$ & A & $\mathrm{k}$ & $\mathrm{c}$ & & $\mathrm{R}^{2}$ & ERM (\%) \\
\hline \multirow{4}{*}{ De difusão } & 55 & 1,080818 & & 0,000018 & & 0,9932 & 3,0318 \\
\hline & 65 & 1,064256 & & 0,000025 & & 0,9945 & 2,6707 \\
\hline & 70 & 1,060819 & & 0,000012 & & 0,9936 & 2,8943 \\
\hline & $\mathrm{T}\left({ }^{\circ} \mathrm{C}\right)$ & $\mathrm{A}$ & $\mathrm{k}_{0}$ & $\mathrm{~b}$ & $\mathrm{k}_{1}$ & $\mathrm{R}^{2}$ & $\operatorname{ERM}(\%)$ \\
\hline \multirow{3}{*}{ Dois termos } & 55 & 0,367218 & 0,008037 & 0,712850 & 0,008038 & 0,9930 & 3,0315 \\
\hline & 65 & 0,392448 & 0,010135 & 0,671221 & 0,010135 & 0,9950 & 2,6705 \\
\hline & 70 & 0,699431 & 0,010470 & 0,361383 & 0,010470 & 0,9940 & 3,6894 \\
\hline \multirow{4}{*}{ Henderson e Pabis } & $\mathrm{T}\left({ }^{\circ} \mathrm{C}\right)$ & $\mathrm{A}$ & $\mathrm{k}$ & & & $\mathrm{R}^{2}$ & ERM (\%) \\
\hline & 55 & 1,080070 & 0,008038 & & & 0,9930 & 3,0315 \\
\hline & 65 & 1,063676 & 0,010135 & & & 0,9950 & 2,6705 \\
\hline & 70 & 1,060815 & 0,010470 & & & 0,9940 & 2,8943 \\
\hline \multirow{4}{*}{$\log$} & $\mathrm{T}\left({ }^{\circ} \mathrm{C}\right)$ & $\mathrm{A}$ & $\mathrm{k}$ & $\mathrm{c}$ & & $\mathrm{R}^{2}$ & ERM $(\%)$ \\
\hline & 55 & 1,108033 & 0,007192 & $-0,04271$ & & 0,9960 & 2,4461 \\
\hline & 65 & 1,086112 & 0,009239 & $-0,03505$ & & 0,9960 & 2,1696 \\
\hline & 70 & 1,085521 & 0,009465 & $-0,03839$ & & 0,9960 & 2,2982 \\
\hline \multirow{4}{*}{ Newton } & $\mathrm{T}\left({ }^{\circ} \mathrm{C}\right)$ & & $\mathrm{k}$ & & & $\mathrm{R}^{2}$ & ERM (\%) \\
\hline & 55 & & 0,007340 & & & 0,9860 & 4,3146 \\
\hline & 65 & & 0,009393 & & & 0,9900 & 3,5857 \\
\hline & 70 & & 0,009741 & & & 0,9900 & 3,6740 \\
\hline \multirow{4}{*}{ Page } & $\mathrm{T}\left({ }^{\circ} \mathrm{C}\right)$ & $\mathrm{K}$ & $\mathrm{n}$ & & & $\mathrm{R}^{2}$ & ERM (\%) \\
\hline & 55 & 0,001729 & 1,292127 & & & 0,9999 & 0,4082 \\
\hline & 65 & 0,003085 & 1,239125 & & & 0,9999 & 0,4237 \\
\hline & 70 & 0,003132 & 1,245417 & & & 0,9998 & 1,4332 \\
\hline \multirow{4}{*}{ Wang } & $\mathrm{T}\left({ }^{\circ} \mathrm{C}\right)$ & $\mathrm{A}$ & $\mathrm{b}$ & & & $\mathrm{R}^{2}$ & ERM $(\%)$ \\
\hline & 55 & $-0,00506$ & 0,000006 & & & 0,9910 & 3,4932 \\
\hline & 65 & $-0,00632$ & 0,000010 & & & 0,9860 & 4,2543 \\
\hline & 70 & $-0,00645$ & 0,000010 & & & 0,9840 & 4,4897 \\
\hline
\end{tabular}


Nas Figuras 2 e 3. pode-se observar que a secagem ocorreu predominantemente no período de taxa decrescente, onde ocorre uma acentuada variação da umidade da amostra em função do tempo, sendo o mecanismo de difusão de vapor responsável pela migração interna da umidade.

A utilização das temperaturas do ar de 65 e $70{ }^{\circ} \mathrm{C}$ resultou em maiores taxas de secagem (Figura 3).
Posteriormente, observa-se menor intensidade da variação da taxa de secagem em função da umidade das amostras, provavelmente devido ao processo passar a ser controlado pelo mecanismo de difusão de vapor do material, na medida em que a água se encontra ligada aos constituintes da polpa.

Figura 2. Cinética da razão de umidade adimensional da polpa de pequi (Y) em função do tempo e da temperatura de secagem. Valores experimentais (símbolo) e estimados pelo modelo de Page (linha contínua).

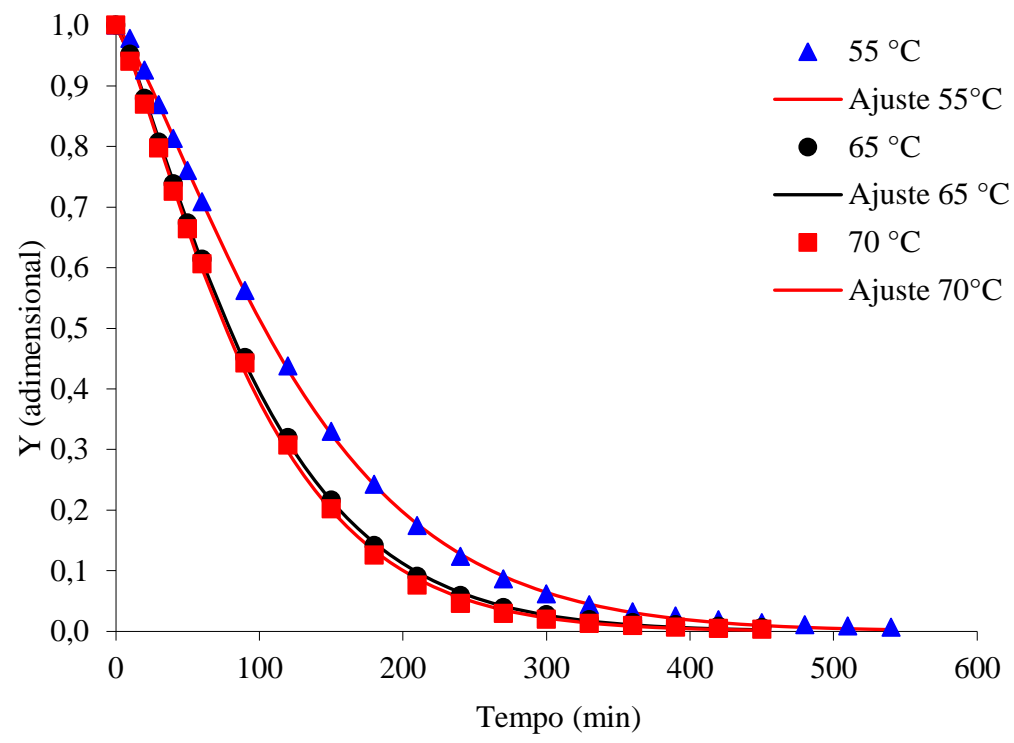

Figura 3. Fluxo de massa do vapor de água em função do conteúdo de umidade da polpa de pequi e da temperatura de secagem.

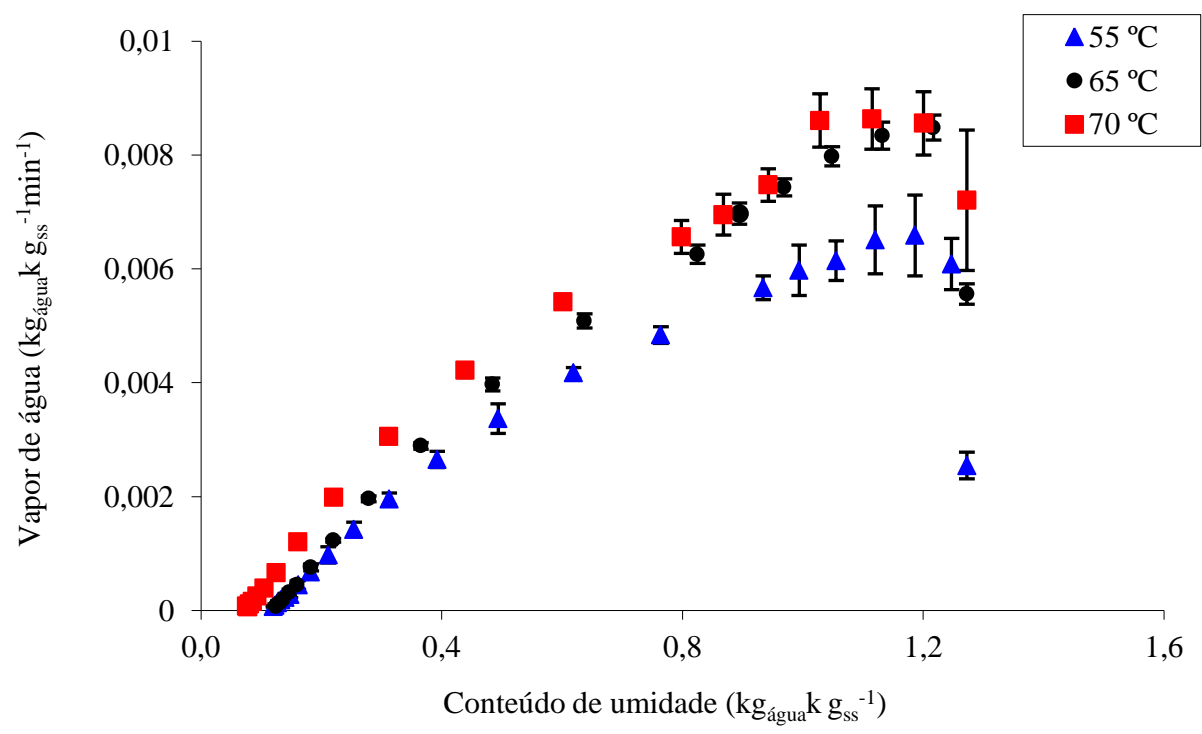

Na Tabela 3. são apresentados os coeficientes de difusividade efetiva $\left(\mathrm{D}_{e f}\right)$. $\mathrm{O}$ aumento da temperatura conduz ao aumento do coeficiente de difusão do vapor da água, acelerando o processo de secagem (GONELI et al., 2007). O aumento da temperatura influenciou na troca térmica entre o ar e a polpa de pequi, os valores de $\mathrm{D}_{e f}$ calculados para a polpa desidratada de pequi encontram-se dentro da faixa de valores do coeficiente de difusão para alimentos, relatada por Chirife (1983) que estão entre $10^{-11}$ a $10^{-9} \mathrm{~m}^{2} \mathrm{~s}^{-1}$.

A partir dos valores da difusividade efetiva foi calculada a energia de ativação (Tabela 3). O valor de energia de ativação apresenta uma grandeza próxima aos valores referenciados por Chirife (1983) para vários produtos alimentícios que é de 1,42 × $10^{4}$ a $3,97 \times 10^{4} \mathrm{~J} \mathrm{~mol}^{-1}$. 
Termodinamicamente a energia de ativação é definida como a facilidade com que as moléculas de água superam a barreira de energia durante e migração do interior do produto (MORAIS et al., 2013). Nos processos de secagem, quanto menor a energia de ativação maior será a difusividade de água do produto. A energia de ativação encontrada neste trabalho foi menor que a obtida por Goneli et al. (2014) na secagem de sementes de folhas de aroeira nas temperaturas de 40, 50, $60 \mathrm{e}$ $70{ }^{\circ} \mathrm{C}$ cujo valor foi de $7,49 \times 10^{4} \mathrm{~J} \mathrm{~mol}^{-1}$.

Tabela 3. Valores da difusividade efetiva $\left(\mathrm{D}_{\mathrm{ef}}\right)$ e Energia de ativação $(\mathrm{Ea})$ para a secagem de polpa de pequi.

\begin{tabular}{ccccc}
\hline $\mathrm{T}\left({ }^{\circ} \mathrm{C}\right)$ & $\mathrm{D}_{\mathrm{ef}}\left(\mathrm{m}^{2} \mathrm{~s}^{-1}\right)$ & $\mathrm{R}^{2}$ & $\mathrm{~T}(\mathrm{~K})$ & $\mathrm{Ea}\left(\mathrm{J} \mathrm{mol}^{-1}\right)$ \\
\hline 55 & $4,04 \times 10^{-10}$ & 0,998 & 328,15 & \\
65 & $5,13 \times 10^{-10}$ & 0,995 & 338,15 & $2,03 \times 10^{4}$ \\
70 & $5,57 \times 10^{-10}$ & 0,997 & 343,15 & \\
\hline
\end{tabular}

Outro estudo por Martins et al. (2015) mostra valores de $8,14 \times 10^{4} \mathrm{~J} \mathrm{~mol}^{-1}$ na secagem de folhas de timbó a 40, 50, 60 e $70{ }^{\circ} \mathrm{C}$. Valores menores que os determinados neste estudo foram relatados por Reis et al. (2011), na secagem da pimenta Cumari do Pará $\left(5,02 \times 10^{3} \mathrm{~J} \mathrm{~mol}^{-1}\right)$ nas temperaturas 45, $55 \mathrm{e}$ $65^{\circ} \mathrm{C}$. Analisando o teor de umidade e a estrutura celular do material, pode-se afirmar que durante a secagem a água pode movimentar-se por diferentes mecanismos no interior do produto. Dependendo do teor de umidade inicial do produto a difusão capilar pode ser o mecanismo predominante. Porém, a complexidade do sistema biológico dos vegetais e o processo de secagem podem também influenciar na velocidade da migração das moléculas de agua, podendo ocorrer difusão líquida, difusão na superfície, difusão de vapor, difusão térmica e fluxo hidrodinâmico (BROOKER et al., 1992)
$\mathrm{Na}$ Tabela 4. são apresentados os valores médios dos componentes nutricionais do pequi fresco e desidratado nas três temperaturas de secagem 55,65 e $70{ }^{\circ} \mathrm{C}$. A umidade inicial da polpa fresca $(54,63 \%)$ foi reduzida a $10 \%$ com a secagem, a evaporação da água contida na polpa favoreceu a concentração de alguns nutrientes. Os teores de umidade entre as farinhas obtidas nas diferentes temperaturas (Tabela 4) não foram alterados pelo período de exposição as temperaturas estudadas. Os valores encontrados de resíduo mineral para polpa fresca e seca foram de $0,01 \mathrm{~g} \mathrm{~g}_{\mathrm{ss}}{ }^{-1}$, corroborando com este estudo Lima et al. (2007) e Santos et al. (2010) que contataram o mesmo valor $\left(0,01 \mathrm{~g} \mathrm{~g}_{\mathrm{ss}}{ }^{-1}\right)$ em polpa in natura e desidratada de pequi.

Tabela 4. Características nutricionais da polpa de pequi fresca e desidratada em função da temperatura de secagem.

\begin{tabular}{|c|c|c|c|c|}
\hline $\begin{array}{r}\text { Constituinte } \\
\end{array}$ & Polpa fresca & Polpa seca $55^{\circ} \mathrm{C}$ & Polpa seca $65^{\circ} \mathrm{C}$ & Polpa seca $70^{\circ} \mathrm{C}$ \\
\hline Umidade $^{* *}\left(g_{\text {água }} 100 g_{\text {amostra }}{ }^{-1}\right)$ & $54,63 \pm 0,24$ & $10,0 \pm 0,05$ & $10,0 \pm 0,05$ & $10,0 \pm 0,11$ \\
\hline Umidade $^{* * * *}\left(\mathrm{~g}_{\text {água }} \mathrm{g}_{\mathrm{ss}}{ }^{-1}\right)$ & $1,20 \pm 0,01$ & $0,10 \pm 0,01$ & $0,10 \pm 0,01$ & $0,11 \pm 0,01$ \\
\hline Resíduo mineral ( $\left.\mathrm{g} \mathrm{g}_{\mathrm{SS}}{ }^{-1}\right)$ & $0,01 \pm 0,02$ & $0,01 \pm 0,05$ & $0,01 \pm 0,15$ & $0,01 \pm 0,05$ \\
\hline Proteínas $\left(\mathrm{g} \mathrm{g} \mathrm{gS}_{\mathrm{S}}^{-1}\right)$ & $0,04 \pm 0,01$ & $0,07 \pm 0,07$ & $0,07 \pm 0,07$ & $0,07 \pm 0,17$ \\
\hline Lipídios $\left(\mathrm{g} \mathrm{g}_{\mathrm{SS}}^{-1}\right)$ & $0,78 \pm 0,06$ & $0,61 \pm 0,12$ & $0,63 \pm 0,04$ & $0,62 \pm 0,14$ \\
\hline Carboidratos $\left(\mathrm{g} \mathrm{gss}^{-1}\right)$ & 0,17 & 0,36 & 0,34 & 0,35 \\
\hline Cor L* & $64,39 \pm 0,2$ & $56,21 \pm 0,18$ & $54,62 \pm 0,38$ & $50,35 \pm 0,23$ \\
\hline Cor a* & $10,07 \pm 0,12$ & $13,00 \pm 0,32$ & $7,34 \pm 0,14$ & $4,14 \pm 0,18$ \\
\hline Cor b* & $42,24 \pm 0,26$ & $29,23 \pm 0,22$ & $27,84 \pm 0,26$ & $17,69 \pm 0,12$ \\
\hline
\end{tabular}

* Média dos valores seguidos pelo desvio padrão \pm ; ** Umidade em base úmida; *** Umidade em base seca

O teor de proteínas concentrou com a desidratação da polpa de pequi. As proteínas geralmente se desnaturam em temperaturas acima de $70^{\circ} \mathrm{C}$ (BADUI, 2006). Porém, de acordo com Epstein e Bloom (2006), as proteínas podem ficar incrustadas na camada dupla de lipídeos o que sugere efeito protetor, diminuindo a degradação das proteínas. Quanto aos lipídios, os valores médios do teor quantificado na polpa fresca são maiores que os encontrados por Arévalo-Pinedo et al. (2010) e Alves et al. (2014) que foi de 0,50 $\mathrm{g} \mathrm{g}_{\mathrm{ss}}{ }^{-1}$, para a mesma espécie em estudo. Na polpa desidratada a 55, 65 e 70 ${ }^{\circ} \mathrm{C}$ o conteúdo de lipídeos diminuiu aproximadamente $25 \%$ em relação à polpa fresca.

No processo de secagem a $70^{\circ} \mathrm{C}$ foi observado o escurecimento da polpa provavelmente devido à reação de Maillard, que ocorre entre açúcares redutores e aminoácidos (PENA et al., 2008). O alto teor de carboidratos (Tabela 4) e a presença de proteínas na polpa desidratada de pequi associados com o aquecimento justificam o escurecimento não enzimático.

Em relação a cor, o decréscimo no valor de $\mathrm{L}^{*}$ indica tendência de escurecimento na polpa provocada pelo tempo de exposição e a temperatura de secagem empregada. Os valores de $\mathrm{b}^{*}$ foram menores na temperatura de $70{ }^{\circ} \mathrm{C}$, mostrando que houve redução da coloração amarelada na polpa desidratada. O processo associado a essa alteração na cor é a degradação dos carotenoides, sobretudo o $\beta$-caroteno. Entre os fatores que interferem na degradação dos carotenoides estão o calor, luz e oxigênio (SANTANA et al., 2013). Diante disto, a secagem a $55^{\circ} \mathrm{C}$ foi considerada a temperatura de processo mais indicada para a obtenção de farinha de pequi por fornecer um produto com melhor aparência visual.

\section{CONCLUSÕES}

O aumento da temperatura de secagem reduz substancialmente o tempo de processo para obter um produto estável em condições ambiente.

O modelo matemático de secagem em camada delgada proposto por Page foi o que melhor se ajustou aos dados experimentais das cinéticas de secagem da polpa de pequi. 
A secagem a $55^{\circ} \mathrm{C}$ por 300 min foi a mais indicada para a obtenção de farinha de pequi com $10 \%$ de umidade, fornecendo um produto com melhor aparência visual.

\section{AGRADECIMENTOS}

Os autores agradecem à Fundação de Apoio ao Desenvolvimento do Ensino, Ciência e Tecnologia do Estado do Mato Grosso do Sul (FUNDECT) e ao Conselho Nacional de Pesquisa e Desenvolvimento $(\mathrm{CNPq})$, pelo suporte financeiro.

\section{REFERÊNCIAS}

ABE, T.; AFZAL, T. M. Thin-layer in infrared radiation drying of rough rice, Journal of Agricultural Engineering Research, v.67, p. 289-297, 1997.

ALVES, A. M.; FERNANDES, D. C.; SOUSA, A. G. de O.; NAVES, R. V.; NAVES, M. M. V. Características físicas e nutricionais de pequis oriundos dos estados de Tocantins, Goiás e Minas Gerais. Brazilian Journal of Food Technology, v.17, n.3, p.198-203, 2014.

ARÉVALO-PINEDO, A.; MACIEL, V. B. V.; CARVALHO, K. M.; COELHO, A. F. S.; GIRALDO-ZUNICÃ, A. D.; ARÉVALO, Z. D. S.; ALVIM, T. C. Processamento e estudo da estabilidade de pasta de pequi (Caryocar brasiliense). Ciência e Tecnologia de Alimentos, v.30, p.664-668, 2010.

AZZOUZ, S.; GUIZANI, A.; JOMAA, W.; BELGHITH, A Moisture difusivity and drying kinetic equation of convective drying of grapes. Journal of Food Engineering, v.55, p.323330, 2002.

BADUI, D. S. Química de los alimentos. 4 ed. México, D. F., Pearson Education, 2006, p.736.

BRASIL. Ministério da Saúde. Agência de Vigilância Sanitária. Resolução $n^{\circ}$ 263, de 22 de setembro de 2005. Aprova o regulamento técnico para produtos de cereais, amidos, farinhas e farelos. Diário Oficial da União; Poder Executivo, 2005.

BROOKER, D. B.; BAKKER-ARKEMA, F. W.; HALL, C. W. Drying and storage of grains and oilseeds. Westport: The AVI Publishing Company, 1992. 450p.

CELMA, A. R.; LÓPEZ-RODRÍGUEZ, F.; BLÁZQUEZ, F. C. Experimental modeling of infrared drying of industrial grape by-products. Food and Bioproducts Processing, v.87, p.247-253, 2009.

CHIRIFE, J. Fundamentals of the drying mechanism during air dehydration of foods. In Advances in food drying (ed.) A. S. Majumdar. New York: Hemisphere Publishing Corporation: 1983, p.73-102.

CRANK, J. The Mathematics of diffusion. $2^{\text {nd }}$ ed. Oxford: Claredon Press, 1975, p.424.
DIAMANTE, L. M.; MUNRO, P.A. Mathematical modelling of hot air drying of sweet potato slices. International Journal of Food Science and Technology, v.26, p.99-109, 1991.

EPSTEIN, E.; BLOOM, A.J. Nutrição mineral de plantas: princípios e perspectivas. Londrina: Editora Planta, 2006.403p.

GONELI, A. L. D.; CORREA, P. C.; RESENDE, O.; REIS NETO, S. A. Estudo da difusão de umidade em grãos de trigo durante a secagem. Ciência e Tecnologia de Alimentos, Campinas, v. 27, p. 135-140, 2007.

GONELI, A. L. D.; VIEIRA, M. do C.; VILHASANTI, H. C. B.; GONÇALVES, A. A. Modelagem matemática e difusividade efetiva de folhas de aroeira durante a secagem. Revista Pesquisa Agropecuária Tropical, v.44, p.56-64, 2014.

HENDERSON, S. M.; PABIS, S. Grain drying theory. II. Temperature effects on drying coefficients. Journal of Agricultural Engineering Research, v.6, p.169-174, 1961.

INSTITUTO ADOLFO LUTZ. Normas analíticas do Instituto Adolfo Lutz. Métodos Físico-Químicos para Análise de Alimentos. 4 ed. São Paulo: Instituto Adolfo Lutz, 2008, p. 1020 .

LIMA, A.; SILVA, A. M. O.; TRINDADE, R. A.; TORRES, R. P.; MANCINI-FILHO, J. Composição química e compostos bioativos presentes na polpa e na amêndoa do pequi (Caryocar brasiliense, Camb.). Revista Brasileira de Fruticultura, v.29, p.695-698, 2007.

LOPES, R. M.; SILVA, J. P.; VIEIRA, R. F.; SILVA, D. B.; GOMES, I. S.; AGOSTINI-COSTA, T. S. Composição de ácidos graxos em polpa de frutas nativas do cerrado. Revista Brasileira de Fruticultura, v.34, p.635-640, 2012.

MACHADO, A. V.; OLIVEIRA, E. L.; SANTOS, E. S.; OLIVEIRA, J. A.; FREITAS, L. M. Estudo cinético da secagem do pendúculo de caju e um secador convencional. Revista Verde de Agroecologia e Desenvolvimento Sustentável, v.5, n.1, p.36-42, 2010.

MARTINS, E. A. S; LAGE, E. Z.; GONELI, A. L. D.; HARTMANN FILHO, C. P.; LOPES, J. G. Cinética de secagem de folhas de timbó. Revista Brasileira de Engenharia Agrícola e Ambiental, v.19, n.3, p. 238-244, 2015.

MORAIS, S. J. da S.; DEVILLA, I. A.; FERREIRA, D. A.; TEIXEIRA, I. R. Modelagem matemática das curvas de secagem e coeficiente de difusão de grãos de feijão-caupi (Vigna unguiculata (L.) Walp.). Revista Ciência Agronômica, 44(3), 455-463, 2013.

MOURA, N. F.; CHAVES, L. J.; NAVES, R. V. Caracterização física de frutos de pequizeiro (Caryocar brasiliense Camb.) do cerrado. Revista Árvore, Viçosa, v.37, n.5, p. 905-912, 2013.

OLIVEIRA, G. H. H. de.; ARAGÃO, D. M. S.; OLIVEIRA, A. P. L. R. de.; SIllVA, M. G.; GUSMÃO, A. C. A. Modelagem e propriedades termodinâmicas na secagem de 
morangos. Brazilian Journal of Food Technology, 18(4), 314$321,2015$.

ÖZDEMIR, M.; DEVRES, Y. O. The thins layer drying characteristics of hazelnuts during roasting. Journal of Food Engineering, v.42, p.225-233, 1999.

PAGE, G. E. (1949) Factors influencing the maximum rates of air drying shelled corn in thin layers. M. Sc. Thesis. Indiana: Purdue University.

PENA, R. S.; SILVA, D. M. S.; MENDONÇA, N. B.; ALMEIDA, M. D. C. Estudo da secagem da fibra residual do maracujá. Revista Brasileira de Tecnologia Agroindustrial, v.2, p.1-13, 2008.

PEREIRA, E. M., SILVA, W. P., GOMES, J. P., SILVA, C. M. D. P. S; FORMIGA, A. S., COSTA, F. B. Empirical models in the description of prickly pear shoot (Nopal) drying kinetics. Revista Brasileira de Engenharia Agrícola e Ambiental, v.21, n.11, p.798-802, 2017.

QUEIROZ, F. A. Impactos da sojicultura de exportação sobre a biodiversidade do cerrado. Sociedade \& Natureza, Uberlândia, v.21, n.2, p.193-209, 2009.

REIS, R. C.; BARBOSA, L. S.; LIMA, M. L.; REIS, J. S.; DEVILLA, I. A.; ASCHERI, D. P. R. Modelagem matemática da secagem da pimenta Cumari do Pará. Revista Brasileira de Engenharia Agrícola e Ambiental, v.15, p.347353, 2011.
SANTANA, A. A.; KUROZAWA, L. E.; OLIVEIRA, R. A.; PARK, K. J. Influence of process conditions on the physicochemical properties of pequi powder produced by spray drying technology, v.31, n.7, p.825-836, 2013.

SANTOS, P.; PORTO, A. G.; SILVA, F. S.; FURTADO, G. F. Avaliação físico-química e sensorial do pequi (Caryocar brasiliensis Camb.) submetido à desidratação. Revista Brasileira de Produtos Agroindustriais, v.12, p.115-123, 2010.

SIQUEIRA, V. C.; RESENDE, O.; CHAVES, T. H. Mathematical modelling of the drying of jatropha fruit: na empirical comparison. Revista Ciência Agronômica, Fortaleza, v.44, n.2, p.278-285, 2013.

SOUZA, E. P.; FIGUEIRÊDO, R. M. F.; GOMES, J. P.; QUEIROZ, A. J. M.; CASTRO, D. S.; LEMOS, D. M. Mathematical modeling of pequi pulp drying and effective difusivity determination. Revista Brasileira de Engenharia Agrícola e Ambiental, v.21, n.7, p.493-498, 2017.

WANG, C. Y.; SINGH, R. P. Use of variable equilibrium moisture content in modeling rice drying. Transaction of ASAE, v.78, p.6505, 1978.

YALDIZ, O.; ERTEKIN, C.; UZUN, H. I. Mathematical modeling of thin layer solar drying of sultana grapes. Energy, v.26, p.457-465, 2001. 